PROCEEDINGS OF THE

AMERICAN MATHEMATICAL SOCIETY

Volume 129, Number 4, Pages 1179-1188

S 0002-9939(00)04940-6

Article electronically published on November 15, 2000

\title{
MARTIN'S AXIOM DOES NOT IMPLY PERFECTLY NORMAL NON-ARCHIMEDEAN SPACES ARE METRIZABLE
}

\author{
YUAN-QING QIAO \\ (Communicated by Andreas Blass)
}

\begin{abstract}
In this paper we prove that in various models of Martin's Axiom there are perfectly normal, non-metrizable non-archimedean spaces of $\aleph_{2}$.
\end{abstract}

A non-archimedean space is defined to be a topological space having a basis for its topology which is a tree under the inclusion relation. We will call a non-metrizable, perfectly normal non-archimedean space an archvillain.

Nyikos [N] asked whether all perfectly normal non-archimedean spaces are metrizable, i.e. do archvillains exist? The only archvillains previously known were Souslin spaces or spaces including Souslin spaces. Todorčević [ $\mathrm{T}_{1}$ proved that in any model of $M A+\neg w K H$, there is no archvillain of weight $\aleph_{1}$. We are going to prove that there are $M A+\neg C H$ models in which there are archvillains essentially of bigger weight. Such models of course have no Souslin spaces.

In $\mathrm{QT}_{1}$, it is proved that every archvillain includes one of size $\aleph_{1}$ if one Mitchell collapses a supercompact cardinal to $\aleph_{2}$. There are Souslin trees in Mitchell models, however, so it is still open whether it is consistent that there are no archvillains.

This work was inspired by conversations with S. Todorčević and is part of the author's doctoral dissertation under the supervision of Prof. F. D. Tall [Q].

We shall use combinatorial principles holding in $L$ to create candidates for archvillains. These candidates are branch spaces of $\kappa$-Souslin trees, where $\kappa$ is either $\aleph_{2}$ or inaccessible in $L$. Then we shall use familiar posets to specialize the initial subtrees of these $\kappa$-Souslin trees to obtain $M A$ models in which these $\kappa$ Souslin trees have become archvillains of weight $\aleph_{2}$.

\section{TERMinOloGy AND NOTATION}

Our topological and set theoretic notations are mainly standard, and can be found in $[\mathrm{E}], \mathrm{K}]$.

Definition. A $\kappa$-Souslin tree is a tree which has height $\kappa$ and has no antichain of size $\kappa$.

Received by the editors December 10, 1992 and, in revised form, January 29, 1998.

1991 Mathematics Subject Classification. Primary 03E05, 54E35, 54B05; Secondary 54G99, 54A35, 03E45.

Key words and phrases. Non-archmedean, perfect, metrizable, tree base, Martin's Axiom, forcing special tree, $\diamond, \square$.

The author's research was supported by NSERC Grant A-7354.

(C)2000 American Mathematical Society 
Definition. A poset is called $\sigma$-dense if any countable intersection of its dense open sets is dense open.

Trees naturally lead to non-archimedean spaces:

Definition. Let $T$ be a tree. Then the branch space of $T$ is $\langle X(T), T\rangle$, where $X(T)=\{b: b$ a branch of $T$ with countable cofinality $\}$ and the basic open sets are determined by the nodes of $b$, i.e. if $t \in b$, then $\left\{b^{\prime} \in T: t \in b^{\prime}\right\}$ is a basic open set about $b$.

Branch spaces are non-archimedean and first countable. Generally, we denote a non-archimedean space by $\langle X, T\rangle$, where $T$ is the topological base. If a nonarchimedean space is first countable, then its points can be thought of as branches of countable cofinality.

Lemma 0.1. An open set in a non-archimedean space is the union of some antichain of the topological tree basis.

Proof. Let $U$ be an open set of $\langle X, T\rangle$. For $t \subset U$ we define $a_{t}$ to be the least member of $\left\{t^{\prime} \in T: t \cap t^{\prime} \neq \emptyset\right.$ and $\left.t^{\prime} \subset U\right\}$. The set $A=\left\{a_{t}: t \in T\right.$ and $\left.t \subset U\right\}$ is an antichain because of the minimality of $a_{t}$ 's. The union of $A$ must be $U$, since for any $x \in U$, there is $t \in T$ such that $x \in t$ and $t \subset U$, hence $a_{t}$ must have been in $A$ already.

We call such an antichain the representative of the open set. We sometimes do not distinguish between an open set and its antichain representative. Also, a point, which is a branch, has a base which is a cofinal chain of that branch. A smaller basic open set is a node at a higher level.

Lemma 0.2. The antichain representative of a dense open set is a maximal antichain.

Proof. If the antichain is extendable, the open set cannot be dense.

Definition. An antichain is called $F_{\sigma}$ if its union is $F_{\sigma}$ in the branch space topology.

Lemma 0.3. If $U=\bigcup_{n \in w} F_{n}$ with the $F_{n}$ 's closed in $X(T)$, then $\widetilde{F}_{n}=\bigcup\left\{a_{\alpha}\right.$ : $\left.a_{\alpha} \cap F_{n}=\emptyset\right\}$ is closed for all $n$, where $\left\{a_{\alpha}: \alpha<\kappa\right\}$ is the representative of $U$.

Proof. If $x$ is not in $\bigcup\left\{a_{\alpha}: a_{\alpha} \cap F_{n} \neq \emptyset\right\}$, then $x$ is not in $F_{n}$, so there is a $t \in T$, $t \cap F_{n}=\emptyset$, such that $x \in t$. This basic open set $t$ does not meet $\widetilde{F}_{n}$, for otherwise, $t$ will meet some $a_{\alpha}$ which meets $F_{n}$. But then either $t \subset a_{\alpha}$ or $t \supset a_{\alpha}$. If $t \subset a_{\alpha}$, then $x$ is in $\bigcup\left\{a_{\alpha}: a_{\alpha} \cap F_{n} \neq \emptyset\right\}$; if $t \supset a_{\alpha}$, then $t$ meets $F_{n}$. This is a contradiction.

Definition. A sequence $W_{1}, W_{2}, \ldots$ of open covers of a topological space is called a development for the space $X$ if for every point $x \in X$ and any neighbourhood $U$ of $x$ there is a natural number $i$ such that $s t(x, W)=\bigcup\left\{w \in W_{i}: x \in w\right\} \subset U . X$ is called developable if it has a development.

A metrizable space is easily seen to be developable.

Lemma 0.4. Non-archimedean spaces are collectionwise normal spaces.

Proof. Let $\langle X, T\rangle$ be a non-archimedean space. If $\left\{F_{i}: i<\kappa\right\}$ is a discrete family of closed sets, then for every $i$ and for every $x \in F_{i}$ there is a $t(x)$ containing $x$ such that $t(x) \cap F_{j}=\emptyset$ for $i \neq j$. Then if $x \in F_{i}, y \in f_{j}, i \neq j$, we have $t(x) \cap t(y)=\emptyset$. 
This can also be seen by using the fact that a non-archimedean space is a generalized ordered space or that it is ultraparacompact [NR].

Lemma 0.5. A branch space $X(T)$ is developable (therefore metrizable, since nonarchimedean spaces are collectionwise normal) if and only if there is a countable family of antichain coverings whose union forms a base.

Proof. If $\left\{A_{1}, A_{2}, \ldots\right\}$ is a sequence of antichain coverings whose union is a basis, then for every point $x$ and any neighbourhood $U$ of $x$, there is an $a(x) \in A_{i}$ for some $i$ such that $x \in a(x)$ and $a(x) \subset U$. Since each $A_{i}$ is an antichain, $\bigcup\left\{a \in A_{i}: x \in a\right\}=a(x)$. Hence such a family is a development.

On the other hand, if $X(T)$ has a development $\left\{W_{1}, W_{2}, \ldots\right\}$, we can replace $W_{i}$ by an antichain covering for all $i$. For every $t \in T$ such that $t \subset \bigcup\left\{w: w \in W_{i}\right\}$, we define $a(t)=\min \left\{t^{\prime} \in T: \exists w \in W_{i}\left(t^{\prime} \cap t \neq \emptyset, t^{\prime} \subset w\right)\right\} . A=\left\{a(t): \exists w \in W_{i}(t \subset\right.$ $w)\}$ is an antichain and it is a refinement of $W_{i}$.

Definition. The upper part above an antichain $A$, denoted as $u p(A)$, is defined as $\{t \in T: \exists a \in A(a \lesseqgtr t)\}$.

Note that the upper part of a maximal antichain $A$ is dense open in a tree $T$, i.e. for any $t \in T$ there is $s \in u p(A)$, such that $t<_{T} s$; and for every $t$, if there is an $s \in u p(A)$ such that $s<t$, then $t$ is in $u p(A)$ as well.

Lemma 0.6. If $T$ is a $\sigma$-dense tree, and the non-archimedean space $(X, T)$ does not have isolated points, then $X$ is not metrizable.

Proof. See Lemma 1.2 in $\mathrm{QT}_{1}$.

Definition. A strong Baire space is a topological space in which the intersection of countably many dense open sets is dense open.

Lemma 0.7. $T$ is $\sigma$-dense if and only if $\langle X, T\rangle$ is strong Baire.

Proof. We first show $\sigma$-density implies strong Baireness. The antichain representative of a dense open set is the maximal. The upper part of the maximal antichain is dense open in the tree $T$. Since the space $X$ is $\sigma$-dense, the intersection of the upper parts of the maximal antichain representatives is dense open in $T$. The union of a dense open set of $T$ is dense open in $X$. Therefore, the intersection of countably many dense open sets of $X$ is dense open.

On the other hand, a maximal antichain of $T$ represents a dense open set. Therefore, strong Baireness implies that the upper parts of countably many maximal antichains will have a dense open intersection.

Lemma 0.8 ([ST]). An archvillain contains an open subspace which is strong Baire.

Proof. See the proof of Theorem 4.2 of [ST], where it is proved that if $X$ is an archvillain, then it contains an open subspace whose base is $\sigma$-dense.

Lemma 0.9. For $\alpha<\mathrm{ht}(T)$ and $\operatorname{cf}(\alpha) \geq \omega_{1}, X(T\lceil\alpha)$ is a subspace of $X(T)$.

Proof. $X\left(T\lceil\alpha) \subset X(T)\right.$, since $\operatorname{cf}(\alpha) \geq \omega_{1}$ and points are branches of countable cofinality. The basic open sets of $X(T\lceil\alpha)$ are of the form $t \cap X(T \uparrow \alpha)$, where $t \in T\lceil\alpha$. 


\section{1. $\aleph_{2}$-SOUSLin TREES AND $\kappa$-SousLin TREeS}

Lemma 1.1. Let $\operatorname{cf}(\kappa)>\omega$. $\kappa$-Souslin trees are $\sigma$-dense.

Proof. A dense open set in a tree is the upper part above a maximal antichain. Since an antichain has size less than $\kappa$ in a $\kappa$-Souslin tree, a maximal antichain must lie in an initial subtree $T \nmid \alpha$ for some $\alpha<\kappa$. Since $\operatorname{cf}(\kappa)>\omega$, given countably many maximal antichains, there will be a $\delta$ such that $\delta<\kappa$ and all of the maximal antichains are included in $T\left\lceil\delta\right.$. For any node $t \in T$, there is a $t^{\prime}$ such that $t<t^{\prime}$ and $t^{\prime}$ is above level $T_{\delta}$, i.e. $t^{\prime}$ is in the intersection of the upper part of the maximal antichains.

$\kappa$-Souslin trees, where $\kappa$ is either $\aleph_{2}$ or inaccessible, will play an important role in our proofs. We need to review the structure of these trees. The notation and techniques used are all from Devlin's book $\mathrm{D}$.

We work in $L$. We are going to construct an $\aleph_{2}$-Souslin tree and a $\kappa$-Souslin tree, where $\kappa$ is an inaccessible cardinal which is not weakly compact. The $L$ machinery employed here is $\square$-sequences and $\diamond$-sequences. The existence of $\square$-sequences at the inaccessible level is assured by the assumption of non-weak compactness.

Definition 1.1. A $\square_{\omega_{1}}(E)$ sequence, where $E \subset \omega_{2}$, is a sequence $\left\{C_{\alpha} \mid \alpha<\omega_{2} \&\right.$ $\lim (\alpha)\}$ such that

(1) $C_{\alpha}$ is club in $\alpha$;

(2) $\operatorname{cf}(\alpha)<\omega_{1} \rightarrow \operatorname{otp}\left(C_{\alpha}\right)<\omega_{1}$;

(3) if $\alpha^{\prime}<\alpha$ is a limit point of $C_{\alpha}$, then $\alpha^{\prime} \notin E$ and $C_{\alpha^{\prime}}=\alpha^{\prime} \cap C_{\alpha}$.

Definition 1.2. A $\diamond_{\omega_{2}}(E)$ sequence is a sequence $\left(S_{\alpha} \mid \alpha \in E\right)$ such that

(1) $S_{\alpha} \subset \alpha$, for all $\alpha$;

(2) whenever $X \subset \omega_{2}$, the set $\left\{\alpha \in E \mid X \cap \alpha=S_{\alpha}\right\}$ is stationary in $\omega_{2}$.

Assume $V=L$. Let $\kappa$ be an inaccessible cardinal which is not weakly compact. Then there is a stationary set $E \subset \kappa$ and a $\square_{\kappa}(E)$ sequence $\left\{C_{\alpha} \mid \alpha<\kappa \& \lim (\alpha)\right\}$ such that

(1) $C_{\alpha}$ is club in $\alpha$;

(2) $\forall \alpha \in E, \operatorname{cf}(\alpha)=\omega$;

(3) if $\alpha^{\prime}<\alpha$ is a limit point of $C_{\alpha}$, then $\alpha^{\prime} \notin E$ and $C_{\alpha^{\prime}}=\alpha^{\prime} \cap C_{\alpha}$.

Also, there is a $\nabla_{\kappa}(E)$ sequence $\left(S_{\alpha} \mid \alpha \in E\right)$ such that

(1) $S_{\alpha} \subset \alpha$, for all $\alpha$;

(2) whenever $X \subset \kappa$, the set $\left\{\alpha \in E \mid X \cap \alpha=S_{\alpha}\right\}$ is stationary in $\kappa$.

We will now construct an $\aleph_{2}$-Souslin tree. The $\diamond_{\omega_{2}}$-sequence is used to capture antichains; the $\square_{\omega_{1}}$-sequence is used in defining those branches as follows:

At level $\alpha, \lim (\alpha)$, for each $x \in T \uparrow \alpha$ we define an $\alpha$-branch $b_{\alpha}^{x}$ of $T\lceil\alpha$ such that $x \in b_{\alpha}^{x}$. If $\operatorname{cf}(\alpha)=\lambda_{\alpha}$, let $\left(\gamma_{\alpha}(\nu) \mid \nu<\lambda_{\alpha}\right)$ be the monotone enumeration of $C_{\alpha}$. We start at the level $T_{\gamma_{\alpha}\left(\nu_{\alpha}(x)\right)}$, where $\nu_{\alpha}(x)$ is the least $\nu$ such that $x \in T\left\lceil\gamma_{\alpha}(\nu)\right.$. Define $p_{\alpha}^{x}\left(\nu_{\alpha}(x)\right)=$ the least ordinal $y \in T_{\gamma_{\alpha}(x)}$ such that $x<_{T} y$. Then we continue to pick up elements at the successor level by $p_{\alpha}^{x}(\nu+1)$, the least $y \in T_{\gamma_{\alpha}(\nu+1)}$, such that $p_{\alpha}^{x}(\nu)<_{T} y$; at the limit level, say $\lim (\eta)$, we pick up $p_{\alpha}^{x}$, namely the unique $y \in T_{\gamma_{\alpha}(\eta)}$ such that $(\forall \nu<\eta)\left(\nu \geq \nu_{\alpha}(x) \rightarrow p_{\alpha}^{x}(\nu)<_{T} y\right)$, provided such a $y$ exists.

Set $b_{\alpha}^{x}=\left\{y \in T\left\lceil\alpha \mid\left(\exists \nu<\lambda_{\alpha}\right)\left(y \leq_{T} p_{\alpha}^{x}(\nu)\right\}\right.\right.$.

The $\square$-sequence prevents the construction of branches from breaking down. We now define $T_{\alpha}$ as follows: 
Case (1). $\alpha \notin E$ or $\alpha \notin$ Lim. We just add new ordinals from $\aleph_{2}$ to provide each branch $b_{\alpha}^{x}, x \in T \uparrow \alpha$, with an extension in $T_{\alpha}$.

Case (2). $\alpha \in E \cap \operatorname{Lim}$, but $S_{\alpha}$ is not a maximal antichain of $T_{\alpha}$. In this case, construct $T_{\alpha}$ just as in Case (1).

Case (3). $\alpha \in E \cap \operatorname{Lim}$, and $S_{\alpha}$ is a maximal antichain of $T \uparrow \alpha$. Then we only extend $b_{\alpha}^{x}$ such that $x$ lies above an element of $S_{\alpha}$.

Such a construction yields an $\aleph_{2}$-Souslin tree. If we use a $\square_{\kappa}$-sequence and a $\nabla_{\kappa}$-sequence, the analogous construction will yield a $\kappa$-Souslin tree, if $\kappa$ is an inaccessible non-weakly compact cardinal $[\mathrm{D}$. We will call Souslin trees constructed in this manner boxy. Note that at any level below $\kappa$, by construction, $T \uparrow \alpha$ has fewer than $\kappa$ cofinal branches.

It is interesting to notice that usually forcing will not add a new chain of cofinality $\omega_{1}$ to these trees. This was proved by Stevo Todorčević $\left[\mathrm{T}_{2}\right]$.

Lemma $1.2\left(\overline{T_{2}}\right)$. Let $M$ be a model of ZFC plus $\diamond_{\aleph_{2}}$ plus $\square_{\aleph_{1}}$ and let $T$ be a boxy $\aleph_{2}$-Souslin tree constructed in $M$. Let $\wp \in M$ be a forcing poset which preserves $\aleph_{1}$, and does not collapse $\aleph_{2}$ to $\aleph_{1}$. Then $T$ will not have new chains of cofinality $\omega_{1}$ in the extension $M^{\wp}$.

Boxy Souslin trees only have branches of countable cofinality, for the underlying set $E$ of the $\nabla_{\kappa}(E)$-sequence is a subset of $\{\alpha \in \kappa: \operatorname{cf}(\alpha)=\omega\}$ and boxy Souslin trees' branches only terminate at level $\alpha, \alpha \in E$.

We note for use in $\mathrm{QT}_{2}$ that the tree we have constructed has at most $\kappa$ many branches of cofinality $\omega$.

Let us fix a boxy $\kappa$-Souslin tree $\mathbf{S}$, and its branch space $X(\mathbf{S})$.

Now we are ready to prove our consistency results.

\section{An MA MODEL With AN ARChVILlain OF WEIGHT $\aleph_{2}$}

To start the forcing process, we choose $L$ as our ground model. We do the usual ccc forcing to obtain $M A+c=\aleph_{2}$ [B]. A modification of the proof in [B] shows that $\wp$ has the $K_{\aleph_{2}}$-property, i.e. any subset of $\wp$ which has size $\aleph_{2}$ has a subset of size $\aleph_{2}$ which is pairwise compatible. By the technique of $[\mathbb{B}$, this implies the forcing will not add new antichains of size $\aleph_{2}$ to $\aleph_{2}$-Souslin trees, in particular, boxy ones. Here is the lemma:

Lemma 2.1. Assume the following:

(a) $P_{\alpha}$ is the direct limit of $\left\langle P_{\beta}: \beta<\alpha\right\rangle$.

(b) If $\beta<\alpha$, then $P_{\beta}$ has size less than $\kappa$.

(c) If $\operatorname{cf} \alpha=\kappa$, then $\left\{\beta<\alpha: P_{\beta}\right.$ is the direct limit of $\left.\left\langle P_{\gamma}: \gamma<\beta\right\rangle\right\}$ is stationary in $\alpha$.

Then $P_{\alpha}$ has the $K_{\kappa}$-property.

Proof. Let $A=\left\{p_{\xi}: \xi<\kappa\right\}$. Let $\left\langle\alpha_{\xi}: \xi<\kappa\right\rangle$ be a continuous sequence of ordinals cofinal in $\alpha$. Define $f: \kappa \rightarrow \kappa$ by $f(\xi)=$ the least $\eta$ such that support $\left(p_{\xi}\left\lceil\alpha_{\xi}\right) \subseteq \alpha_{\eta}\right.$. (c) implies $f$ is regressive on a stationary subset of $\kappa$. By the pressing down lemma, $f$ is constant on a stationary set $S$, i.e. $f^{\prime \prime} S=\{\eta\}$. Since $P_{\alpha_{\eta}}$ has size less than $\kappa,\left\{p_{\xi}\left\lceil\alpha_{\eta}: \xi \in S\right\}\right.$ has property $K_{\kappa}$. By thinning out, we can assume that if $\xi_{1}, \xi_{2} \in S$, then $p_{\xi_{1}}$ and $p_{\xi_{2}}$ are compatible, and support $\left(p_{\xi_{1}}\right) \subseteq \alpha_{\xi_{2}}$. Let $r \leq p_{\xi_{1}} \uparrow \alpha_{\eta}, p_{\xi_{2}}\left\lceil\alpha_{\eta}\right.$, and define $q \in P_{\alpha}$ by $q\left\lceil\alpha_{\eta}=r\right.$ and $q(\beta)=p_{\xi_{1}}(\beta)$, if $\alpha_{\eta} \leq \beta<\alpha_{\xi_{2}}$; or $p_{\xi_{2}}(\beta)$, if $\alpha_{\xi_{2}} \leq \beta<\alpha$. Then $q \leq p_{\xi_{1}}, p_{\xi_{2}}$. So $\left\{p_{\xi}: \xi \in S\right\}$ are pairwise compatible. 
Since $S$ remains an $\aleph_{2}$-Souslin tree in the extension, it is therefore $\sigma$-dense, and therefore the branch space $X(S)$ is not developable.

It will suffice to prove $X(T)$ is perfect, i.e. open sets of $X(S)$ are $F_{\sigma}$, since non-archimedean spaces are normal.

To see that $X(S)$ is perfect in the extension we need two lemmas as follows:

Definition. $T$ is called an $\aleph_{1}$-tree if $\operatorname{ht}(T)=\omega_{1}$ and $|T|=\aleph_{1}$.

An $\aleph_{1}$-tree may have uncountable levels.

Lemma 2.2. The usual poset (see e.g., [B]) which specializes an $\aleph_{1}$-tree which has no cofinal branches actually produces a generic development of the ground model branch space of this tree.

Proof. This is a density argument. The conditions of the poset are finite pieces of a countable family of antichains of the tree, i.e. $p \in \wp$ if and only if $|p|<\aleph_{0}$, $\operatorname{dom}(p) \subset T, \operatorname{rang}(p) \subset \omega$ and for all $n, p^{-1}(n)$ is an antichain. It suffices to prove that the generic antichains are actually coverings of the space, for the union of the generic antichains is the whole tree and therefore a development.

To see that each antichain of the family is a covering, we just need to know that for any point of the space, we can always add one basic open set to the antichain to conclude this point is in the union of the antichain.

For $x \in X(T), n<\omega$, let $D_{x, n}=\{p \in \wp: \exists t \in T(p(t)=n$ and $x \in t)\}$. We verify $D_{x, n}$ is dense:

If $p$ is not in $D_{x, n}$, then $x$ is not in any $t$ such that $p(t)=n$. Since $p$ is finite, there will be some $t^{\prime} \in T$ such that $x \in t^{\prime}$ and for $t \in \operatorname{dom}(p), p(t)=n$ implies $t \cap t^{\prime}=\emptyset$. Let $q\left(t^{\prime}\right)=n$ and for $t \in \operatorname{dom}(p), q(t)=p(t)$. Then $q$ extends $p$.

In the extension, $T=\bigcup\left\{A_{n}: n<\omega\right\}$, where each $A_{n}$ is an antichain cover of the space. For $x \in X, \operatorname{st}\left(x, A_{n}\right)$ is the unique $t \in A_{n}$ such that $x \in t$.

Lemma 2.3. If an $\aleph_{1}$ tree $T$ has at most $\aleph_{1}$ many cofinal branches, its branch space has a basis $T^{\prime}, T^{\prime} \subset T$, such that $T^{\prime}$ is an $\aleph_{1}$-tree with no $\omega_{1}$-branches.

Proof. Enumerate cofinal branches by $\left\{b_{\alpha}: \alpha<\omega_{1}\right\}$. Let $t_{0}$ be the least element of $b_{0}$, let $t_{\alpha}$ be the least element of $b_{\alpha} \backslash \bigcup\left\{b_{\beta}: \beta<\alpha\right\}$, let $S=\left\{t: \exists \alpha\left(t_{\alpha}<t \in b_{\alpha}\right)\right\}$, and let $T^{\prime}=T \backslash$.

$T^{\prime}$ is a basis of $X(T)$. Otherwise, there would be a point $x$ which would be a branch of countable cofinality whose tail is a subset of $S$, i.e. there is an $\alpha$ such that $x \backslash x\left\lceil\alpha \subset S\right.$. Let $\beta_{0}$ be the least $\beta$ such that $b_{\beta} \cap\left(x \backslash x\lceil\alpha) \neq \emptyset\right.$. But $x \backslash b_{\beta_{0}}$ is not empty, for otherwise $x$ cannot be a branch of countable cofinality. Therefore there is a least element $t$ of $x \backslash b_{\beta_{0}} ; t$ is in $S$, hence there is a $b_{\beta}$ which contains $t$. Let $\beta_{1}$ be the minimal such $\beta$. Clearly, $\beta_{0}<\beta_{1}$. Then $x \cap b_{\beta_{0}} \subset \bigcup\left\{b_{\beta}: \beta<\beta_{1}\right\}$, so $t_{\beta_{1}}>x \cap b_{\beta_{0}}$. But $t_{\beta_{1}} \leq t$, so $t_{\beta_{1}}$ is in $x \backslash x \mid \alpha$. This is a contradiction.

We call a tree base trimmed if all its branches of uncountable cofinality are deleted but it remains a base. Therefore Lemma 2.3 provides a way of trimming an $\aleph_{1}$-tree which has at most $\aleph_{1}$ many $\omega_{1}$-branches.

Applying Lemma 2.3 inductively, we can trim trees of size $\aleph_{1}$ with at most $\aleph_{1}$ many branches of cofinality $\omega_{1}$.

Lemma 2.4. If $T$ is a tree of size $\aleph_{1}$ with at most $\aleph_{1}$ many branches of uncountable cofinality, then there is a subset $T^{\prime}$ of $T$, which is an $\aleph_{1}$-tree with no $\aleph_{1}$-branches, and $T^{\prime}$ is a base of $X(T)$. 
Proof. Let $\left\{\lambda_{\alpha}: \alpha<\omega_{2}\right\}$ be the increasing continuous sequence of ordinals which either have cofinality $\omega_{1}$ and are less than $\omega_{2}$, or are $\omega$-limits of such ordinals.

We prove the lemma by induction on the height of trees.

The case $\lambda_{0}=\omega_{1}$, i.e. $\operatorname{ht}(T)=\omega_{1}$, is covered by Lemma 2.3

Now we consider the successor case, $\lambda_{\alpha+1}$. $T \uparrow \lambda_{\alpha}$ has been trimmed. This is just another tree of height $\omega_{1}$. So we trim $T \backslash T\left\lceil\lambda_{\alpha}\right.$. The union of these two trimmed parts, $T\left\lceil\lambda_{\alpha} \cup\left(T \backslash T\left\lceil\lambda_{\alpha}\right)^{\prime}\right.\right.$, is a base of $X(T)$.

Finally, we consider the limit cases, either $\operatorname{cf}(\alpha)=\omega_{0}$ or $\operatorname{cf}(\alpha)=\omega_{1}$.

In the first case, we pick $\lambda_{\alpha_{1}}<\lambda_{\alpha_{2}}<\cdots<\lambda_{\alpha_{n}}<\cdots<\lambda_{\alpha}$. Each $T\left\lceil\lambda_{\alpha_{n}}\right.$ can be trimmed. Without loss of generality, we assume each $T \uparrow \lambda_{\alpha_{n}}$ has been already trimmed. Therefore, each $T \uparrow \lambda_{\alpha_{n+1}} \backslash T \uparrow \lambda_{\alpha_{n}}$ has been trimmed. We keep the bottom level of each $T \nmid \lambda_{\alpha_{n+1}} \backslash T \uparrow \lambda_{\alpha_{n}}$. This will not change the topology of the corresponding branch spaces, but this step is necessary to ensure that the cofinal branches of $T\left\lceil\lambda_{\alpha}\right.$ have cofinal chains in the direct sum of trimmed $T\left\lceil\lambda_{\alpha_{n+1}} \backslash T \uparrow\right.$ $\lambda_{\alpha_{n}}$ 's.

In the second case, we fix an increasing sequence $\lambda_{\alpha_{1}}<\lambda_{\alpha_{2}}<\cdots<\lambda_{\alpha_{\beta}}<\cdots<$ $\lambda_{\alpha_{\omega_{1}}}$, where $\alpha_{\omega_{1}}=\alpha$. We trim each $T \nmid \lambda_{\alpha_{\beta+1}} \backslash T \uparrow \lambda_{\alpha_{\beta}}$ with its bottom level intact. Then take the direct sum. Now we will get an $\omega_{1}$-tree which may still have some $\omega_{1}$-branches. Apply Lemma 2.3 once more, and it is done.

Also, by the proof of Lemma 2.2. since as is well known the specializing poset satisfies the countable chain condition, we get the following result:

Lemma 2.5 $(M A)$. If $(X, T)$ is first countable, non-archimedean, $|X|<2^{\omega}, T$ has at most $\aleph_{1}$ many branches of uncountable cofinality, then $X$ is metrizable.

We now return to the proof of perfectness. In the extension, $M A$ holds. Therefore all branch spaces of $\aleph_{1}$-trees with at most $\aleph_{1}$ many cofinal branches are metrizable, according to Lemma 2.5 By Lemma 1.2, we know that the boxy $\aleph_{2}$-Souslin tree has no new initial branches of cofinality $\aleph_{1}$, hence for all $\alpha$ with $\operatorname{cf}(\alpha)=\aleph_{1}, X(S\lceil\alpha)$ has a development. Any open set $U$ in $X(S)$ is actually a union of an antichain of $S$. Let $a=\left\{a_{\alpha}: \alpha<\omega_{1}\right\}$ be the antichain. We are going to expand this antichain of size $\aleph_{1}$ to an $\aleph_{1}$ subtree of $S$. Pick one point, a branch of countable cofinality, $x_{\alpha}$ from $a_{\alpha}, \forall \alpha<\omega_{1}$. There is some $\eta<\aleph_{2}$ such that $\left\{x_{\alpha}: \alpha<\omega_{1}\right\} \subset S\left\lceil\eta\right.$ and $\operatorname{cf}(\eta)=\omega_{1}$. By the lemmas, we conclude that the branch space of $S\lceil\eta$ is metrizable, and therefore the open set $U \cap X(S\lceil\eta)$ in question is $F_{\sigma}$ in the subspace $X(S \uparrow \eta) . U=\bigcup F_{n}$ with the $F_{n}$ 's closed in $X(S \uparrow \eta)$ and we know by Lemma 0.3 that we can assume $F_{n}=\bigcup\left\{a_{\alpha} \cap X(S \uparrow \eta): a_{\alpha} \cap F_{n}=\emptyset\right\}$. But then, it is easy to see $U$ is also $F_{\sigma}$ in $X(S)$. Let $\widetilde{F}_{n}=\bigcup\left\{a_{\alpha}: a_{\alpha} \cap F_{n} \neq \emptyset\right\}$; then some $\widetilde{F}_{n}$ would be not closed, if $U$ were not $F_{\sigma}$. There would be a point $x \in \operatorname{cl}\left(\widetilde{F}_{n_{0}}\right) \backslash \widetilde{F}_{n_{0}} . x$ is not in $X(S \uparrow \eta)$, by Lemma 0.3 Hence this $x$ must lie above $S_{\eta}$, hence there would be a $t \in S_{\eta}$ such that $x \in t$. Since $x$ is a limit point of $\widetilde{F}_{n_{0}}$, $t$ intersects $\widetilde{F}_{n_{0}}$. Therefore there is $a_{\alpha}, a_{\alpha} \cap \widetilde{F}_{n_{0}} \neq \emptyset$, and $t$ meets $a_{\alpha}$. But, then $t \subset \widetilde{F}_{n_{0}}$, a contradiction.

The weight of $X(S)$ is $\aleph_{2}$. If $X$ has a base $B$ of size $\aleph_{1}$, for each $b \in B$, find one $t_{b} \in S$ such that $t_{b} \subset b$. $T^{\prime}=\left\{t_{b}: b \in B\right\}$ is included in $S\lceil\alpha$ for some $\alpha<\aleph_{2}$. Clearly $T^{\prime}$ does not include a base for points which are branches of countable cofinality in $S \backslash S \uparrow \alpha$. Now, we can claim the following theorem:

Theorem 1. Con $\left(M A+c=\aleph_{2}+\right.$ there is a non-metrizable, perfectly normal nonarchimedean space of weight and size $\aleph_{2}$ ). 
There has been some interest in constructing non-metrizable spaces such that small subspaces are metrizable. Here we have a perfectly normal one. To see that every subspace of size $\aleph_{1}$ is metrizable, we only need to point out that for a subspace of size $\aleph_{1}$, there must be some $\alpha$ such that the subspace is included in the branch space of $T \uparrow \alpha$.

\section{AN $M A$ MODEL WITH AN ARCHVILLAin OF WEIGHT AND SIZE $\aleph_{2}$ BUT NONE OF SIZE $\aleph_{1}$}

Since the boxy $\aleph_{2}$-Souslin tree gains no new $\omega_{1}$ initial branches, it will yield an archvillain of weight $\aleph_{2}$ in any $M A$ model created by a property- $K_{\aleph_{2}}$ forcing which does not collapse $\aleph_{1}$. In [ST], Shelah and Todorčević obtained an $M A$ model with no Baire space of size $\aleph_{1}$. Noticing that by Lemma 0.8 an archvillain contains an open subspace which is Baire, we see that this model contains no archvillains of size $\aleph_{1}$. We can prove that in this model there is an archvillain of weight and size $\aleph_{2}$ by verifying the forcing they used has property $K_{\aleph_{2}}$.

The poset used in $[\mathrm{ST}]$ is a countable support iteration of $\aleph_{2}$ steps. All ccc posets of size $\aleph_{1}$ appear in the iteration, in particular $\wp_{\omega_{2}}$ forces $M A_{\aleph_{1}}$ plus $2^{\aleph_{0}}=\aleph_{2}$. The iteration takes care of $\aleph_{1}$-trees as follows:

For any $\wp_{\omega_{2}}$-name $\dot{T}$ of a tree of size $\aleph_{1}$ and height $\omega_{1}$ there exist stationarily many $\delta<\omega_{2}$ with $\operatorname{cf}(\delta)=\omega_{1}$ such that $\wp_{\delta+1}=\wp_{\delta} * \dot{C}_{\omega_{1}} * \wp_{T} * S_{T^{*}}$, where $\dot{C}_{\omega_{1}}$ is the standard poset for adding $\aleph_{1}$ Cohen reals, $\dot{C}_{\omega_{1}} * \wp_{T}$ is a poset for embedding a generic tree $T^{*}$ into $T$ and $\dot{S}_{T^{*}}$ is a ccc poset for specializing $T^{*}$ ST.

The iteration has the following properties:

1. $\forall \alpha<\omega_{2}, \wp_{\alpha}$ satisfies $\aleph_{2}$-p.i.c. ( $\aleph_{2}$ properness isomorphism condition) [ST].

2. $\dot{C}_{\omega_{1}} * \wp_{T} * S_{T^{*}}$ satisfies $\aleph_{2}$-p.i.c.

3. $\wp_{\omega_{2}}$ is proper and $\aleph_{2}$-cc $([\mathrm{ST}],[\mathrm{S}])$.

4. $\dot{C}_{\omega_{1}} * \wp_{T} * S_{T} *$ satisfies $\aleph_{2}$-p.i.c. $[\mathrm{S}]$.

$\mathrm{CH}$ is preserved at all intermediate stages since we have the following lemma.

Lemma 3.1 ([S] $)$. If $\alpha_{0}<\kappa,\left\langle P_{\alpha}, Q_{\alpha}: \alpha<\alpha_{0}\right\rangle$ is a countable support iteration, each $Q_{\alpha}$ satisfies the $\kappa$-p.i.c. ${ }^{*}$, and $(\forall \mu<\kappa) \mu^{\aleph_{0}}<\kappa$, then $2^{\aleph_{0}}<\kappa$ in $V^{P_{\alpha_{0}}}$.

Now we are ready to prove that $\wp_{\omega_{2}}$ has property $K_{\aleph_{2}}$. First we examine the poset $\wp_{T}$.

Let $T$ be a tree of size $\aleph_{1}$ and height $\omega_{1}$. Let $B_{T}$ be the set of all $\omega_{1}$-branches of $T$. Let $\wp_{T}$ be the set of all pairs $p=\left\langle\pi_{p}, f_{p}\right\rangle$ where:

1) $\pi_{p}$ is an order and level preserving map from a countable initial part of $T$ onto a countable initial part of $\left(\omega_{1}\right)^{<\omega_{1}}$;

2) $f_{p}$ is a countable partial map from $B_{T}$ into $\omega_{1}$ such that every member of $\operatorname{dom}\left(f_{p}\right)$ intersects each level of $\operatorname{dom}\left(\pi_{p}\right)$;

$3)$ if $f_{p}(b)=f_{p}(c)$, then $\pi_{p}(s)=\pi_{p}(t)$ for all $s \in b \cap \operatorname{dom}\left(\pi_{p}\right)$ and $t \in c \cap \operatorname{dom}\left(\pi_{p}\right)$ of the same height.

For $p, q \in \wp_{T}$, define $q \leq p$ if and only if $\pi_{q} \supseteq \pi_{p}, f_{q} \supseteq f_{p}$ and $\pi_{p}(s) \neq \pi_{q}(t)$ for all $s \in \operatorname{dom}\left(\pi_{p}\right)$ and $t \in \operatorname{dom}\left(\pi_{q} \backslash \pi_{p}\right)$.

Since $\mathrm{CH}$ holds at all intermediate stages of the iteration, the size of the set $\left\{\pi_{p}: p \in \wp_{T}\right\}$ is forced to be at most $\aleph_{1}$. By the same reason so are the Cohen forcing poset and the poset which specializes an $\aleph_{1}$-tree. The iteration satisfies the $\aleph_{2}$ properness isomorphism condition $[\mathrm{ST}]$ and therefore is $\aleph_{2}$-c.c. In fact as we 
shall show, by a minor variation of the proof of $\aleph_{2}$-c.c. in $[\mathrm{S}], \aleph_{2}$-p.i.c. implies the $K_{\aleph_{2}}$ property, if $C H$ holds in the ground model.

Definition. $\wp$ satisfies the $\kappa$-p.i.c. ( $\kappa$ properness isomorphism condition) if the following holds:

If $\lambda$ is big enough, $\left\langle N_{i}: i<\kappa\right\rangle$, is a sequence, where

1) $\forall i, N_{i}$ is a countable elementary submodel of $\langle H(\lambda),<, \in\rangle$;

2) $\forall i<\kappa, \kappa \in N_{i}, \wp \in N_{i}, i \in N_{i}$;

3) $\forall i<j, N_{i} \cap \kappa \subset j, N_{i} \cap i=N_{j} \cap j$, and $h$ is an isomorphism from $N_{i}$ onto $N_{j}$, $h \uparrow\left(N_{i} \cap N_{j}\right)=i d, h(i)=j$;

then for any $i$, any $p \in N_{i}$, there is $q \in \wp$, such that:

a) $q \leq p, h(p)$, and for every maximal antichain $A \subset \wp, A \in N_{i}, A \cap N_{i}$ is predense below $q$;

b) for every $r \in N_{i}$ and $q^{*}, q^{*} \geq q$, there is $q^{\prime \prime} \leq q^{*}$ such that $\left[q^{\prime \prime} \leq r\right.$ iff $q^{\prime \prime} \leq$ $h(r)]$.

Lemma 3.2. Suppose $\left(\forall \mu<\aleph_{2}\right) \mu^{\aleph_{0}}<\aleph_{2}$, and $\wp$ satisfies the $\aleph_{2}$-p.i.c. Then $\wp$ satisfies the $K_{\aleph_{2}}$-property.

Proof. Let $p_{i} \in \wp, i<\aleph_{2}$. Let $A=\left\{p_{i}: i<\aleph_{2}\right\}$ and let $\lambda$ be a sufficiently large cardinal. Let, for all $i \in \aleph_{2}, i \in N_{i}, p_{i} \in N_{i}, A \in N_{i}, N_{i} \prec H(\lambda)$, and $N_{i}$ countable. Let $h(i)=\sup \left(N_{i} \cap i\right)$, so $h$ is a regressive function. By Fodor's lemma, for some $\gamma,\{i: h(i)=\gamma\}$ is stationary.

By $C H$, there is some $B \subset \gamma, S=\left\{i: N_{i} \cap i=B\right\}$ is stationary and we can take a $B^{\prime} \supset B$ such that $i \neq j \in S \Rightarrow N_{i} \cap N_{j}=B^{\prime}$. Now there are $\aleph_{2}$ models $\left(N_{i}, p_{i}, i, b\right)_{b \in B}$ and $\aleph_{2}>2^{\aleph_{0}}$, and the number of isomorphism types of such models is $2^{\aleph_{0}}$, so for $\aleph_{2}$ many $i$ 's, $N_{i}$ have the same isomorphism type. So for these models, if $i<j$ there is an isomorphism $h: N_{i} \rightarrow J_{j}($ onto $), h\left(p_{i}\right)=p_{j}, h\left\lceil B^{\prime}=i d\right.$. Now apply the definition of $\aleph_{2}$-p.i.c. By item (a), $p_{i}$ and $h\left(p_{i}\right)=p_{j}($ since $h(i)=j)$ are compatible. Hence, $\wp$ has $K_{\aleph_{2}}$-property.

Corollary $(\mathrm{CH})$. The poset $\wp_{\omega_{2}}$ has property $K_{\aleph_{2}}$.

Theorem 2. If $T$ is a boxy $\aleph_{2}$-Souslin tree and $V$ is a model of $C H$, then in $V^{\wp \omega_{2}}$ :

1) $M A+2^{\aleph_{0}}=\aleph_{2}$;

2) there is no archvillain of size $\aleph_{1}$;

3) there is an archvillain of weight and size $\aleph_{2}$.

\section{AN $M A$ MOdel With an ARCHVILlain OF Weight $\aleph_{2}$ AND NONE OF WEIGHT $\aleph_{1}$}

This time, we are going to prove that when we carry out the usual forcing $\mathrm{B}$ to collapse an inaccessible $\kappa$ to $\omega_{2}$ to get $C O N(M A+\neg w K H)$, if $\kappa$ is not weakly compact, then $\mathbf{S}$, a boxy $\kappa$-Souslin tree, becomes a $\aleph_{2}$-Souslin tree and its branch space $X(\mathbf{S})$ becomes perfectly normal.

The forcing iteration we use is described in Baumgartner's paper [B]. Recall $\neg w K H$ asserts that every $\aleph_{1}$-tree has at most $\aleph_{1}$ cofinal branches. We set $L$ as our ground model and $\kappa$ as an inaccessible non-weakly compact cardinal in $L$; at each step of the iteration, we use a product of three posets: the first one is a ccc poset (for the sake of obtaining $M A$ ), the second is a countably closed poset (collapsing a cardinal below $\kappa$ ), the third one specialize an $\aleph_{1}$-tree (in order to specialize all 
$\aleph_{1}$-trees in the extension). The iteration has countable support, i.e. full support at stages of countable cofinality and finite support elsewhere.

Notice that all the posets we used in the iteration have size less than $\kappa$, so the iteration has the $K_{\kappa}$-property by Lemma 2.1] A property $K_{\kappa}$ forcing will not add any new $\kappa$ antichain to $\mathbf{S}[\mathbf{B}$, hence $\mathbf{S}$ remains a $\kappa$-Souslin tree in the extension in which $\kappa$ has been collapsed to $\aleph_{2}$.

Theorem 3. $\operatorname{Con}(\exists$ inaccessible $)$ implies $\operatorname{Con}(M A+$ "all non-archimedean spaces of weight $\leq \aleph_{1}$ are metrizable" + "there is a non-archimedean space of weight $\aleph_{2}$ which is perfectly normal but not metrizable").

Proof. Similar to the proof of Theorem 1, start with a $\kappa$-Souslin tree where $\kappa$ is inaccessible but not weakly compact. Note e.g. the least inaccessible is not weakly compact. Then we use proper forcing to get an $M A$ model in which there is no $\aleph_{1}$-tree with more than $\aleph_{1}$ uncountable branches. By $\left[\mathrm{T}_{1}\right]$, all perfectly normal non-archimedean spaces of weight $\leq \aleph_{1}$ are metrizable.

In conclusion, I thank the referee for many helpful comments.

\section{REFERENCES}

[B] J. E. Baumgartner, Iterated Forcing, London Mathematical Society Lecture Note Series 87, Cambridge Univ. Press, 1978. MR 87c:03099

[D] K. J. Devlin, Constructibility, Springer-Verlag, 1984. MR 85k:03001

[E] R. Engelking, General Topology, Helderman Verlag, Berlin, 1989. MR 91c:54001

[K] K. Kunen, Set Theory, North Holland, 1980. MR 82f:03001

[N] P. Nyikos, A survey of Zero-Dimensional Spaces, Topology (Proc. Nineth Annual Spring Conf., Memphis State Univ., Memphis, Tenn., 1975), pp. 87-114. MR 56:1245

[NR] P. J. Nyikos and H. C. Reichel, On the structure of zero-dimensional spaces, Indag. Math. 37 (1975), 120-136. MR 51:1779

[P] S. Purisch, The orderability of nonarchimedean spaces, Top. and its Appl. 16 (1983), 273277. MR 85f:54063

[Q] Y. Q. Qiao, On Non-archimedean Spaces, Thesis, Univ. of Toronto, 1992.

$\left[\mathrm{QT}_{1}\right]$ Y. Q. Qiao and F. D. Tall, Perfectly normal non-archimedean spaces in Mitchell models, Top. Proc. 18 (1993), 231-243. MR 96g:54033

$\left[\mathrm{QT}_{2}\right]$ Y. Q. Qiao and F. D. Tall, Perfectly normal non-metrizable non-archimedean spaces are generalized Souslin lines, Proc. Amer. Math. Soc. (to appear).

[S] S. Shelah, Proper Forcing, Springer-Verlag Lecture Notes in Mathematics, 940, 1982. MR 84h:03002

[ST] S. Shelah and S. B. Todorčević, A note on the small Baire spaces, Canad. J. Math. 38 no. 3 (1986), 659-665. MR 88c:54005

$\left[\mathrm{T}_{1}\right]$ S. B. Todorčević, Some consequences of $M A+\neg w K H$, Topology and its Appl. 12 (1981), 203-220. MR 83b:03060

$\left[\mathrm{T}_{2}\right]$ S. B. Todorčević, Trees, subtrees and order types, Ann. Math. Logic 20 (1981), 233-268. MR 82m:03062

Department of Mathematics, University of Toronto, Toronto, Canada M5S 3G3 